use of attenuated pathogens is the use of commensal bacteria as vaccine carriers ${ }^{7}$. Several theoretical objections have been raised to the use of commensal organisms in vaccines, most importantly the potential induction of immune tolerance following longterm exposure to target antigens continually released by organisms that colonize mucosal surfaces. Such induction of immune tolerance has not been observed experimentally.

In one example, Medaglini et al. ${ }^{8}$ engineered the human oral commensal Streptococcus gordonii to express on its surface a 204-amino acid allergen from hornet venom as a fusion with the M6 protein of Streptococcus pyogenes. Both systemic and mucosal antibody responses against the allergen were detected in mice orally colonized with this recombinant Gram-positive commensal carrier.

In the present paper, Robinson and colleagues have taken this concept one step further using $L$. lactis as the vaccine carrier. Previous studies from their laboratory have demonstrated that mice inoculated parenterally or intranasally with $L$. lactis expressing fragment $C$ of tetanus toxin (TTFC) develop systemic serum antibodies against tetanus toxin that protects against lethal challenge ${ }^{9,10}$. The current work extends this concept to oral immunization with $L$. lactis expressing TTFC. Immunization via the oral route resulted in increased TTFC-specific serum IgG and fecal IgA and protection against lethal toxin challenge. An intriguing additional finding is that formalin or mitomycin $C$ killed L. lactis are as effective as live $L$. lactis in eliciting serum IgG anti-TTFC when administered intranasally.

One drawback to the use of $L$. lactis as a carrier for oral immunization is the number of doses required ( 7 intragastric inoculations with $5 \times 10^{9}$ cells each in the current study). TTFC is a highly immunogenic protein and less immunogenic proteins may require higher doses or more immunizations. There was also no indication that the T-helper (Th) 1 arm of the immune response was activated when TTFC was expressed by L. lactis, this is in contrast to with the findings of Yamamoto et al. ${ }^{11}$ in which TTFC expressed in an attenuated S. typhimurium elicited both ThI (interferon- $\gamma$ and interleukin-2) and Th2 (interleukin-10, but not interleukin-4 or interleukin-5) cytokine responses.

In vaccine development, as in life, there are no singular solutions. Vaccine strategies that elicit cell-mediated immunity may be preferred for some infectious diseases. In such cases, attenuated Salmonella spp. may be the best choice as a vaccine carrier. Other infectious diseases may benefit from primarily humoral responses, in which case a commensal streptococcus or Gram-positive bacterium such as $L$. lactis may be a better choice of carrier. The use of these bacteria as delivery vectors of heterologous antigens to the secretory immune system constitutes a promising approach for the development of new vaccines against a number of diseases. Even though there are unresolved questions about the use of this technique, the results obtained to date are encouraging, and there is great potential here for development of safe, effective, affordable vaccines.

1. Robinson, K., Chamberiain, L.M., Schofield, K.M., Wells, J.M., and LePage, R.W.F. 1997. Nature Biotechnology 15:653-657.
2. Càrdenas, L. and Clements, J.D. 1992. Clin. Microbiol. Rev. 5:328-342.

3. Curtiss, R. et al. 1994. Dev. Biol. Stand. 82:23-33

4. Levine, M.M. et al. 1996. J. Biotechnol. 44:193-196.

5. Cirillo, J.D., Stover, C.K., Bloom, B.R., Jacobs, W.R., and Barletta, R.G. 1995. Clin. Infect. Dis. 20:1001-1009.

6. Renauld-Mongenie, G. et al. 1996. Proc. Natl. Acad. Sci. USA 93:7944-7949.

7. Fischetti, V.A., Medaglini, D., and Pozzi, G. 1996. Curr. Opin. Biotechnol. 7:659-666.

8. Medaglini, D., Pozzi, G., King, T.P., and Fischetti, V.A. 1995. Proc. Natl. Acad. Sci. USA 2:6868-6872.

9. Wells, J.M., Wilson, P.W., Norton, P.M., Gasson, M.J., and LePage, R.W.F. 1993. Mol. Microbiol. 8:1155-1162

10. Wells, J.M., Norton, P.M., and LePage, R.W.F 1995. Int. Dairy J. 5:1071-1079.

11. Yamamoto M., et al. 1996. Ann. N.Y. Acad. Sci. 778:64-71.

\title{
Entering the domain of neurotrophin binding
}

\section{Barbara L. Hempstead and Moses V. Chao}

The neurotrophins are a family of four structurally related peptide growth factors that regulate neuronal differentiation, survival, and synaptic transmission of both peripheral and central neurons. Their pivotal role in nerve survival has suggested they would be useful in the treatment of many types of neurodegenerative diseases; however, clinical therapeutic trials of these proteins have been disappointing, complicated by the relative difficulties of delivery and pharmacokinetics in the central nervous system. Now, the identification of neurotrophin receptor domains reported in this issue by Holden et al. ' throws some light on the mechanism of receptor binding and provides a necessary first step for the eventual identification of therapeutically useful peptide mimetics of these proteins.

Nerve growth factor (NGF), brainderived neurotrophic factor (BDNF), neurotrophin-3 (NT-3) and neurotrophin 4/5 (NT-4/5) comprise the neurotrophin family -a group of highly homologous, highly basic, peptide growth factors that circulate as homodimers. They regulate neuronal function by interacting with members of the family of Trk receptor tyrosine kinases, which have in their extracellular domains cysteinerich and leucine-rich regions, and more membrane proximal immunoglobulin-like domains ${ }^{2}$. Each neurotrophin demonstrates selectivity of Trk activation, with NGF binding to Trk A, BDNF and NT-4/5 binding to

Barbara L. Hempstead is associate professor of medicine and Moses $V$. Chao is professor of cell biology in the departments of medicine and cell biology, Cornell University Medical College, New York, NY 10021

(blhempst@mail.med.cornell.edu).
Trk B, and NT-3 binding most avidly to Trk C. A second class of neurotrophin receptor, with distant homology to the tumor necrosis factor (TNF) family of receptors has also been identified. This receptor, p75, binds all neurotrophins, but lacks an inherent enzymatic activity.

Although some classes of neurons exhibit marked fidelity in Trk receptor expression, and thus neurotrophin responsiveness, other subsets of neurons can express multiple Trk receptors, or may exhibit different patterns of Trk receptor expression at distinctive developmental stages. Furthermore, the identity of cells that locally synthesize neurotrophins in the adult central nervous system (CNS) is still under intense investigation. Although some neurotrophin synthesis is target derived, local autocrine production by neurons, as well as paracrine production by astrocytes and microglia, has been hypothesized.

Several different approaches have identified immunoglobulin-like domains of the Trk A receptor tyrosine kinase as those critical for NGF binding. In the present study, Holden et al. demonstrate that coinjection of purified TrkA immunoglobulin-like domains with NGF prevents the biological response of local skin edema formation normally elicited upon NGF intradermal injection. These results confirm previous in vitro binding studies that mapped the immunoglobulin domains as the critical regions for Trk-neurotrophin interaction ${ }^{3-5}$. The utilization of an immunoglobulin-like domain for peptide growth factor binding appears to be a recurrent theme in ligand--receptor signaling by receptor tyrosine kinases, as the fibroblast growth factor receptors and the vascular endothelial 
growth factor receptor Flk-1 utilize related immunoglobulin domains for the binding of their respective ligands $\mathrm{s}^{6,7}$.

Their studies also compliment the identification of the critical amino acids of the neurotrophins that are required not only for neurotrophin-Trk binding, but also to convey specificity of Trk activation ${ }^{8}$. The crystal structure of NGF has been solved, and models of neurotrophin-Trk interactions have been proposed ${ }^{9}$. Thus, mutation of specific residues of NGF can render it ineffective in binding to all Trks, whereas directed mutagenesis of other amino acids of NT-3 can result in a "pan-neurotrophin" that can effectively bind and activate all three classes of Trk receptor ${ }^{8,10}$. The data from these previous reports, together with the current work of Holden and coworkers should produce a windfall of information for researchers in structure-based drug design and for predicting peptides capable of activating Trk receptors.

Besides the IgC2-like domains, the region between the transmembrane and the $\operatorname{IgG}$ domain nearest to the membrane may also be critical for binding of neurotrophins $s^{4,11}$. Another extracellular motif, the second leucine-rich repeat, has been reported to be sufficient for binding to NGF in the absence of the IgG domain ${ }^{12}$. Although there is a debate about the relative importance of the IgG domain versus the leucine-rich repeat, the differences may lie in the type of biochemical assay and the state of the recombinant proteins. However, the results suggest there is a potential for multiple interactions between receptor and neurotrophin, which is dependent upon the conformational status of each protein. Indeed, contacts between NGF and Trk A are present in the $\beta$-hairpin loops, and the amino and carboxyl termini of NGF. The intrinsic flexibility of these regions suggests that conformational changes may occur on formation of a productive NGF-Trk complex?.

Unlike many other ligand-receptor systems that utilize highly limited patterns of tissue expression to convey selectivity of action, the neurotrophins and their receptors are highly expressed in contiguous regions of the brain. These observations suggest that the selectivity of neurotrophin action may be regulated in the specific affinities of ligand -receptor interaction. Therefore, the accuracy in identifying the appropriate binding domains of Trk receptors that convey selectivity of neurotrophin binding will be critical for the eventual development of "docking" peptides that utilize a common Trk binding site, but display selectivity in activation of only a single class of Trk receptor.

Further studies of this kind will address the issue of what residues determine neurotrophin specificity. The current findings open the prospect of mimicking neurotrophins with small molecules that may display therapeutic potential in diseases ranging from Alzheimer's disease and amyotrophic lateral sclerosis to the ameloriation of pain pathways. Drug design and modeling are now within the realm of reality, as the regions responsible for neurotophin-receptor binding are now being defined to a small and accessible domain.

1. Holden, P.H., et al. 1997. Nature Biotechnology
15:668-672.

2. Lewin, G. and Barde, Y.-A. 1996. Annu. Rev. Neurosci. 19:289-317.

3. Perez, P. et al. 1995. Mol. Cell. Neurosci. 6:97-105.

4. Urfer, R. et al. 1995. EMBO J. 14:2795-2805.

5. Tannahill, L., Klein, R., and Schachner, M. 1995. Eur. J. Neurosci. 7:1424-1428.

6. Blechman, J.M. et al. 1995. Cell 80:103-113.

7. Davis-Smyth, T., Chen H., Park, J. et al. 1996. EMBO J. 15:4919-4927.

8. Ibanez, C.F. 1995.Trends Biotechnol. 13:217-227.

9. McDonald, N.Q. and Chao, M.V. 1995. J. Biol. Chem 270:19669-19672.

10. Urier, R. et al. 1994. EMBO J. 13:5896-5909.

11. MacDonald, J.I. and Meakin, S.O. 1996. Mol. Cell. Neurosci. 7;371-390.

12. Windisch, J.M., Steiner, M.R., and Schneider, R.J. 1995. Biol. Chem. 270:28133-28138.

\section{Evolution goes for GOLD in silico}

\section{Clare Sansom}

Several techniques call on evolution to discover or adapt potential drug molecules. Now, similar evolutionary principles are being applied in silico. GOLD-genetic optimization for ligand docking-(Jones et al., J. Mol. Biol. 267:727-748, 1997) is a program developed by Gareth Jones at the University of Sheffield (Sheffield, UK) in collaboration with Glaxo Wellcome (London) and the Cambridge Crystallographic Data Centre (CCDC; Cambridge, UK) where the technique is applied to the problem of docking ligands to protein binding sites.

Accurate ligand docking, typically of actual or potential drug molecules, to proteins is one of the most important unsolved problems in computer-aided drug design. If a ligand is flexible, picking its single active bound conformation out of the huge range of possible conformations is an immensely complex and time-consuming problem. The traditional docking approach predicts the structure and binding free energy of a ligand-receptor complex using the structures of the free ligand and receptor. With the development of docking approaches that scan hundreds of three-dimensional structures in public databases, the need for more efficient algorithms has become more pressing.

This type of problem-searching a manydimensional conformational space for a small subset of possible solutions-is an ideal application for a genetic algorithm. These use simulated Darwinian evolution to obtain rapid and accurate solutions to complex searches.

Clare Sansom is a freelance writer working in Reading, UK.
A typical run of GOLD takes 30 minutes to a few hours (depending on the flexibility of the ligand) much less than the computationally demanding conformational searching programs. GOLD represents each protein-ligand complex as a set of "chromosomes" ("artificial creatures" composed simply of strings of characters). These are subjected to random "mutations" and "crossovers"-analogous to the respective processes in sexual reproduction - to produce daughter chromosomes which are tested against a fairly standard energy function. Eventually, an initial random population of chromosomes will converge onto a set of the "fittest" structures-which should, in theory, correspond to the optimum conformation of the protein-ligand complex.

GOLD has passed the classic test of docking algorithmspredicting the conform- ational "flip" between methotrexate and folate docked to dihydrofolate reductase-with flying colors. In a much more rigorous test, Robin Taylor at the CCDC used it to predict the binding conformations of an unbiased set of 100 complexes taken from the Protein Data Bank (PDB; Brookhaven, NY). The results were compared against the actual conformations and assessed subjectively as "good"; "close"; "significant errors"; or "just plain wrong". Counting the first two classes as "successes", the program now has a success rate of $75 \%$, as good as commercially available knowledge-based or conformational search methods. Although it is unlikely that this will be repeated in a real drug design situation, as drug molecules show more structural diversity than ligands found in the PDB, GOLDis still likely to become a very useful tool. 